Portland State University

PDXScholar

Fall 2021

\title{
Understanding the Effect of Dietary Palmitic Acid on Glycolysis During Innate Immune Memory in Macrophages
}

Khaleda A. Aqaei

Portland State University

Follow this and additional works at: https://pdxscholar.library.pdx.edu/honorstheses

Part of the Immunopathology Commons, Medical Cell Biology Commons, and the Medical Immunology Commons

Let us know how access to this document benefits you.

\section{Recommended Citation}

Aqaei, Khaleda A., "Understanding the Effect of Dietary Palmitic Acid on Glycolysis During Innate Immune Memory in Macrophages" (2021). University Honors Theses. Paper 1154.

https://doi.org/10.15760/honors.1193

This Thesis is brought to you for free and open access. It has been accepted for inclusion in University Honors Theses by an authorized administrator of PDXScholar. Please contact us if we can make this document more accessible: pdxscholar@pdx.edu. 
TITLE: Understanding the effect of dietary palmitic acid on glycolysis during innate immune memory in macrophages

\author{
By \\ Khaleda Aqaei
}

An undergraduate honors thesis submitted in partial fulfillment of the requirements for the degree of
Bachelor of Science
in
University Honors
and
Biology

\author{
Thesis Advisor \\ Brooke Ann Napier, Ph.D. \\ Assistant Professor Biology Department
}

Portland State University

Fall, 2021 


\title{
Portland State University \\ PDXScholar
}

\section{University Honors Thesis}

$11 / 22 / 21$

TITLE: Understanding the effect of dietary palmitic acid on glycolysis during innate immune memory in macrophages

\author{
AUTHORS: Khaleda Aqaei and Brooke A. Napier \\ Biology Department, Portland State University
}

\section{A. ABSTRACT:}

Trained immunity is long-term innate immune memory induced by a primary stimulus, which leads to hyper-inflammation upon secondary stimulation with a homologous or heterologous ligand. Trained immunity is mediated by epigenetic and metabolic reprogramming of the target cell and leads to modification of gene expression and cellular function. Classically, trained immunity is initiated by $\beta$-glucans, an inflammatory molecule found on the exterior of fungal species. Interestingly, our lab has recently described that dietary fatty acids can initiate trained immunity, working through similar pathways as $\beta$ glucans. Specifically, our data show that a pre-treatment with a specific dietary saturated fatty acid (SFA), palmitic acid (PA), initiates trained immunity in macrophages, leading to a hyper-inflammatory response to a secondary challenge with numerous microbial ligands. Macrophages are tissue-specific innate immune cells that play a key role in orchestrating inflammatory diseases and infection. Though we have reported PAdependent trained immunity leads to a hyper-inflammatory response, it is unknown if PA induces metabolic changes associated with induction of trained immunity. Specifically, enhanced glycolysis occurs during the trained immune response. Thus, the goal of this study is to determine if PA enhances glycolysis, like classical stimulants of trained immunity, during enhanced inflammation upon secondary stimulation with LPS. We aim 
to quantify the expression of three key glycolytic genes within mouse macrophages with PA-induced trained immunity: 1) glut1, which allows glucose to enter the cell, and 2) hk2 and 3) pfkp, which encode for two rate-limiting enzymes in glycolysis. We hypothesized that PA induces glycolysis during trained immunity in macrophages. We found that after $24 \mathrm{~h}$ of treatment with PA followed by a secondary heterologous stimulus, PA induced expression of glut1, but not $h k 2$ or pfkp. Additionally, if we treated macrophages with PA for $24 \mathrm{~h}$, rested it for $24 \mathrm{~h}$, and then added a secondary stimulus, there was no longer a significant increase in expression of $h k 2$. Our results indicate that PA may enhance glycolysis during the trained immune response, however if the macrophage is rested for $24 \mathrm{~h}$ after exposure to $\mathrm{PA}$, it no longer induces a glycolytic response. These data highlight the dynamic glycolytic response of the macrophage during PA-dependent trained immunity. The capacity for PA to directly impact innate immune metabolism associated with inflammatory pathways may inform dietary interventions and treatments for patients with acute or chronic inflammatory diseases.

\section{B. BACKGROUND:}

The vertebrate immune system has traditionally been divided into innate and adaptive arms. Innate immunity is the branch of the immune system that responds rapidly to infection and is activated within minutes of detecting non-self or infections. The initial innate immune response is required to initiate the adaptive immune response, which requires several days to mount an effective humoral (antibody driven) and cellular response (T-cell driven) (Netea. et al., 2011). Each of these branches of the immune system have specific cell types to carry out immunological functions required for responding to and clearing and infectious agent.

Monocytes are innate immune cells that originate in the bone marrow and migrate throughout the body to sites of infection, where they differentiate into tissue-resident macrophages. Macrophages orchestrate inflammation in response to infection, and help clear infection by immediately responding to, engulfing, and destroying pathogens Arango (Duque, et al., 2014, Martin, et al., 2014). Macrophages express pattern recognition receptors (PRRs) on their cell membrane and within the cell that allow them to recognize 
specific pathogen-associated molecular patterns (PAMPs) found on many pathogens. One example of a PAMP is the molecule lipopolysaccharide (LPS), which is found on the outer membrane of many Gram-negative bacterial species (Matsuura, 2013). The recognition of LPS by PRRs on macrophages leads to inflammation and is considered a non-specific feature of innate immunity, because many different species of Gramnegative bacteria can be recognized this way (Netea, el al., 2020). When a macrophage detects LPS, it rapidly induces an inflammatory response, which is mediated by the production and secretion of signaling molecules called cytokines. Cytokines are synthesized by the macrophage and work in an autocrine, paracrine, and endocrine manner to alert other innate and adaptive immune cells of an invasion by a pathogen (Arango Duque and Descoteaux, 2014; Murphy, Weaver, Janeway, 2017, Janeway's Immunobiology).

In contrast, adaptive immune cells identify specific proteins called antigens that are unique to only one species of pathogen and use antigens to develop antibodies and cellular immunity that is built to recognize and neutralize one specific pathogen. Additionally, adaptive immunity can mount a memory response to this one specific pathogen (Netea, et al., 2011); and, it has been thought that only adaptive immune cells have the ability to remember pathogens.

Historically, it was believed that innate immune cells did not retain memory of pathogens following infection; however, it has been recently appreciated that innate immunity has nonspecific memory that is regulated by different mechanisms than adaptive immune memory (Netea, et al., 2011). In 2012, Netea's group found macrophages are capable of organizing a memory response called trained immunity (Netea, et al., 2013), and it is mediated by distinct metabolic and epigenetic alterations that have not been fully characterized (Netea, et al., 2020).

Trained Immunity: Trained immunity is an adaptation of the innate host defense system that allows macrophages to develop non-specific, and long-lived memory to a group of pathogens (Divangahi \& Netea, et al., 2020). This memory phenomenon has been shown to occur in both in vitro (cell culture) and in vivo model systems of mice, as well as humans in clinical settings (Netea, et al., 2011). Specifically, $\beta$-glucans have been demonstrated 
to induce enhanced inflammatory signaling capacity within human monocytes and macrophages via metabolic and epigenetic alterations (Dominguez-Andres, et al., 2019). Similarly, the Bacillus Calmette-Guérin (BCG) vaccine, the vaccine against Mycobacterium tuberculosis infection, is known to enhance monocyte and macrophage responses to secondary infection with unrelated pathogens in human patients (Kleinnijenhuis, 2011). The impacts of BCG- and $\beta$-glucan-induced trained immunity within human monocytes and macrophages involve a well-known metabolic feature called the Warburg Effect, whereby enhanced aerobic glycolysis allows for the activated cell to meet the energy demands necessary for enhanced inflammatory cytokine production and secretion in response to infection (Warburg, 1923; EM Palsson-Mcdermott, O'Neill et al., 2015, Cell Metabolism; Cheng, et al., 2014, Science).

Interestingly, trained immunity has also been shown to be a feature of macrophages that have been exposed to non-microbial stimuli or "sterile" triggers. For example, atherosclerotic mice that have been fed a Western Diet (WD), a diet enriched in saturated fats and sugar, exhibit a long-lasting reprogramming of innate immune cells with enhanced inflammatory capacity (Christ et al., 2019). Human macrophages have also been shown to exhibit a long-lasting enhanced inflammatory response to LPS following brief exposure to oxidized low-density lipoprotein, (oxLDL is a molecule leads to epigenetic reprogramming of monocytes, eventually causing long-term elevated proinflammatory cytokine production), to induce trained immunity (Bekkering et al., 2014). Recently, the mechanisms of trained immunity induced by sterile triggers have been shown to involve metabolic alterations that remodel the epigenetic landscape of human and mouse monocytes and macrophages (Bekkering, et al., 2018, Cell; DominguezAndres et al., 2019; Saeed, et al., 2014). While these studies have identified key metabolic pathways, including glycolysis, that play important roles in trained immunity, no one has shown the impact of dietary constituents on these pathways in macrophages. Specifically, saturated fatty acids (SFAs) are known to have immunomodulatory impacts on innate immune cells and alter inflammatory responses (Napier, et al., 2019). However, the mechanisms underlying the capacity of SFA to modulate metabolism within macrophages during a trained immunity response has not been explored. 
Palmitic Acid and Trained Immunity: Palmitic acid (PA) is a long-chain saturated fatty acid that is found in diets enriched in animal fats including meat, milk, and butter, (Carta, et al., 2017). Many groups have shown that PA can induce inflammation in macrophages and monocytes, (Napier et al., 2019, Korbecki, et al. 2019, Laine, et al., 2007, Tzeng, et al., 2019, Schwartz, et al., 2010).

Our group has collected preliminary data that demonstrates macrophages treated with a physiologically relevant concentration of PA $(1 \mathrm{mM})$ for $24 \mathrm{~h}$, and then stimulated with a secondary heterologous inflammatory stimulus (LPS $10 \mathrm{ng} / \mathrm{mL}$ ) show a significantly higher production of key inflammatory cytokines (TNF, IL-6, and IL-1 $\beta$ ) (Fig 1A-C), compared to macrophages treated with LPS alone. These are the first data that suggest that PA can induce trained immunity in macrophages, and currently we are actively understanding the mechanisms behind this immune regulation.

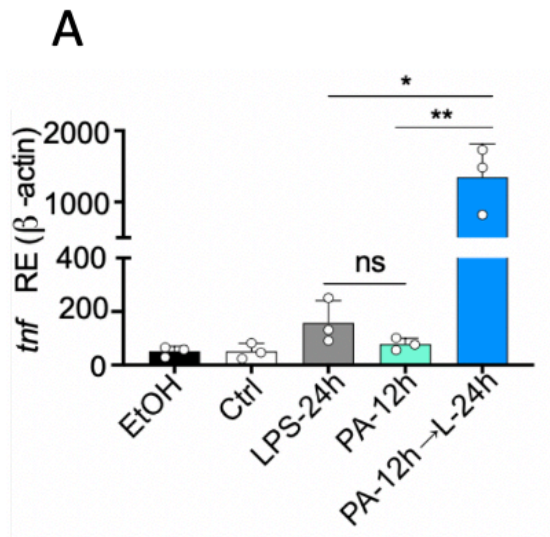

B

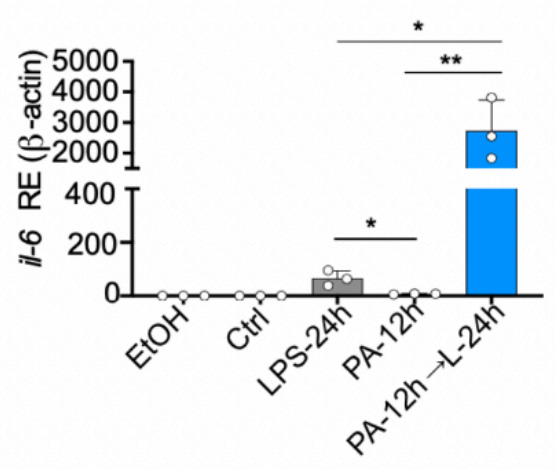

C

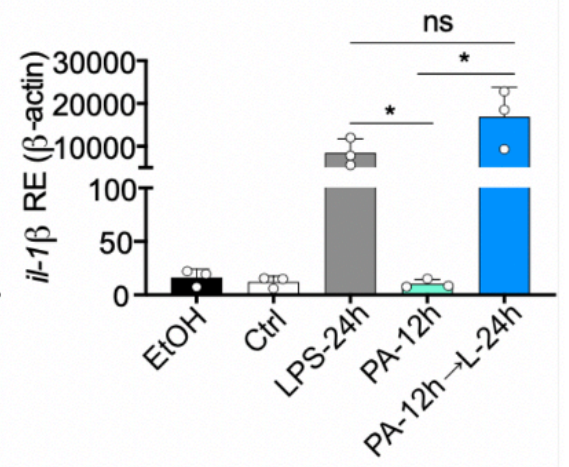

Figure 1. Palmitic acid induces a hyper-inflammatory response to secondary challenge with LPS in macrophages. Primary bone marrow-derived macrophages (BMDMs) were isolated from aged-matched females and males. A-C BMDMs were plated at $1 \times 10^{6} \mathrm{cells} / \mathrm{mL}$ and treated with either ethanol (EtOH; media with $1.69 \%$ ethanol), media (LPS Ctrl; media only), or LPS $(100 \mathrm{ng} / \mathrm{mL})$ for 12 or $24 \mathrm{~h}$. Next, BMDMs were treated with either EtOH, media, LPS $(10 \mathrm{ng} / \mathrm{mL})$ for $24 \mathrm{~h}$, PA $(500 \mu \mathrm{M}$; diluted in $0.8 \%$ EtOH) for $12 \mathrm{~h}$ with or without secondary challenges with LPS. After indicated time points, RNA was isolated and expression of a TNF, $\mathbf{b}$ il- 6 , $\mathbf{c}$ il- $1 \beta$ was measured via qRT-PCR for all plates, all treatments were performed in triplicate. For all panels, a student's $t$-test was used for statistical significance. ${ }^{*} p<0.05 ;{ }^{* *} p<0.01$; ${ }^{* * *} p<0.001$. For all panels, error bars show the mean $\pm \mathrm{SD}$. (Seufert, et al. in preparation). 
Inflammation and Glycolysis: As mentioned previously, monocytes and macrophages orchestrate the production and secretion of inflammatory cytokines during infection and inflammatory disease. Inflammation is initiated when monocytes and macrophages detect danger- or pathogen- associated molecular patterns (DAMPs and PAMPs) via plasma membrane associated pattern recognition receptors (PRRs). Once PRRs identify a DAMP or PAMP, this initiates metabolic reprogramming that is required for the increased production of inflammatory cytokines (Trine H. Mogensen, 2009). For example, when primary macrophages are treated with LPS it is detected by a PRR called TLR4. After detection of LPS by TLR4, TLR4 is activated and induces glycolysis and increased synthesis of pro-inflammatory cytokines (Yang et al., 2014). Additionally, this metabolic reprogramming is essential for the anti-microbial functions of activated macrophages, termed M1 macrophages (Zhu et al., 2015). M1 macrophages require an increase in glucose consumption and lactate release during inflammation, whereas non-inflammatory macrophages (M2 macrophages) or resting macrophages mainly employ oxidative phosphorylation (Zhu et al., 2015). The metabolic change in M1 macrophages is called the Warburg effect (aerobic glycolysis), which has been extensively characterized in tumor cells, and is associated with an increase in glucose uptake and an elevated rate of glycolysis (Palsson-McDermott et al., 2013).

Furthermore, in M1 macrophages, the transcription factor HIF1 $\alpha$ is upregulated which increases the expression of genes encoding for glycolytic enzymes, as well as other inflammatory mediators (Viola et al., 2019). This creates a positive feedback loop - further enhancing inflammation and glycolysis. One of the glycolytic genes regulated by HIF1a is glut1 (Viola et al., 2019). Studies have revealed that the upregulation of glycolytic enzyme GLUT1 is important for the glycolytic activity of M1 macrophages as it facilitates rapid glucose uptake required for the production of inflammatory cytokines (Viola et al., 2019). Another enzyme regulated by HIF1a is 6-phosphofructo-2-kinase B (PFKFB) (Viola et al., 2019). M1 macrophages express primarily the PFKFB3 isoform which, if compared to the other isoforms, less efficiently catalyzes the conversion of fructose-2,6bisphosphate in fructose 6-phosphate, thus enhancing the glycolytic flux (Viola et al., 
2019). Together, after detection of a DAMP or PAMP, M1 macrophages up-regulate glycolysis and inflammatory cytokines, and specifically up-regulated transcription factors, like HIF1a, that play an important role in regulating the expression of genes encoding for glycolytic enzymes.

\section{AIM:}

The goal of this study is to determine if PA enhances glycolysis during the PA-induced trained immunity response and if so, does the glycolysis persist long-term. Specifically, we aim to 1) quantify the expression of three key glycolytic genes within mouse macrophages treated with PA, followed by LPS, and 2) quantify the expression of three key glycolytic genes within mouse macrophages treated with PA, then rested for $24 \mathrm{~h}$, followed by LPS. We hypothesize that PA induces glycolysis in macrophages during PA-induced trained immunity.

\section{APPROACH:}

Summary: We used an in vitro model of mouse macrophages to simulate PA-induced trained immunity using PA as the first inflammatory stimulus and lipopolysaccharide (LPS) as the secondary, heterologous inflammatory stimulus. Mouse bone marrow-derived macrophages (BMDMs) were extracted from the femurs of mice, cultured, and frozen back, as described previously (Seufert et al., 2021, in preparation). Next, we 1) treated BMDMs with PA for 24 hours (h) and treated with LPS for $24 \mathrm{~h}$ or we 2) treated BMDMs with PA for 24 hours (h), rested for 1 day in fresh media and treated with LPS for $24 \mathrm{~h}$. After treating the cells, we isolated the mRNA from the cell lysates and determined the expression of genes that code for glucose transport and glycolytic enzymes (Schematic of workflow: Fig 2).

Extraction and treating of BMDM: Mouse bone marrow-derived macrophages (BMDMs) were isolated from aged-matched female and male wild-type C57BL/6 mice. Mice were euthanized and femurs were dissected and centrifuged to collect bone marrow. Bone 
marrow was resuspended in DMEM supplemented with M-CSF, and BMDMs were cultured for 6 days prior to storage at $-80^{\circ} \mathrm{C}$ with $90 \%$ FBS and $10 \%$ DMSO. Next, BMDMs were thawed and cultured for 5 days, and then collected, pelleted, washed, and resuspended in media (DMEM) containing 10\% FBS, $2 \%$ bovine serum albumin (BSA; ProLiant Biologicals), and 10\% M-CSF. In order to initiate PA-induced trained immunity, we first plated cells at $2.5 \times 10^{5}$ cells/well in 24-well tissue culture plates and treated them with either ethanol (EtOH; media with 1.69\% ethanol), media (Naive Ctrl; media only), or palmitic acid (1mM; diluted in 1.69\% EtOH, Sigma-Aldrich) for $24 \mathrm{~h}$. This concentration of PA was used because it reflects the concentration of PA found in the blood of obese humans (Korbecki, et al., 2019). After the 24h PA treatment, all media was removed from the wells, and the cells rested for 1 day in fresh media to explore for memory, after one day if the cells epigenetically modify and respond to inflammation by enhancing the trained immunity.

Next, we either immediately added LPS as the secondary, heterologous stimulus to induce hyper-inflammation in macrophages or, rested the cells for $24 \mathrm{~h}$ and then treated with LPS for $24 \mathrm{~h}$. Specifically, cells were treated with $10 \mathrm{ng} / \mathrm{mL}$ LPS (Ultrapure LPS, E. coli 0111: B4, Invivogen) and incubated at $37^{\circ}$ for an additional $24 \mathrm{~h}$. After indicated time points, mRNA was isolated and expression of glycolysis genes (glut1, $h k 2$, and pfkp) were measured via qRT-PCR, as discussed below.

RNA extraction and real-time qRT-PCR: To assess the expression of glycolytic genes, we first isolated mRNA from treated BMDMs. First, we lysed the BMDMs with trizol in the fume hood then we flash froze in the liquid nitrogen for 20 seconds and stored them within $-80^{\circ} \mathrm{C}$. Next, we continued mRNA extractions using RNeasy Mini Kit (Qiagen), following the manufacturer's instructions. Then, we created cDNA from mRNA samples using the SuperScript III First-Strand synthesis system (Invitrogen).

Next, we used previously published gene-specific primers that target glycolytic genes of interest (Liu et al., 2020, Cell Host \& Microbes). [glut1 Forward: TCAACACGGCCTTCACTG; glut1 Reverse: CACGATGCTCAGATAGGACATC; hk2 Forward: TGATCGCCTGCTTATTCACGG; hk2 Reverse: AACCGCCTAGAAATCTCCAGA; pfkp Forward: CGCCTATCCGAAGTACCTGGA; pfkp 
Reverse: CCCCGTGTAGATTCCCATGC] Using these gene-specific primers we amplified transcripts using FastStart Universal SYBR Green Master (BioRad). Importantly, we had 4 types of treatments in this experiment: 1 ) naive (+/- rest), 2) naive + LPS, 3) PA (+/-) rest and 4) LPS and PA ctrl, and we calculated relative expression of glycolytic genes to the housekeeping gene, [ $\beta$-actin Forward: CTGTCCCTGTATGCCTCTG; $\beta$-actin Reverse: ATGTCACGCACGATTTCC]. Additionally, each experiment described within this section was repeated in biological and experimental triplicate.

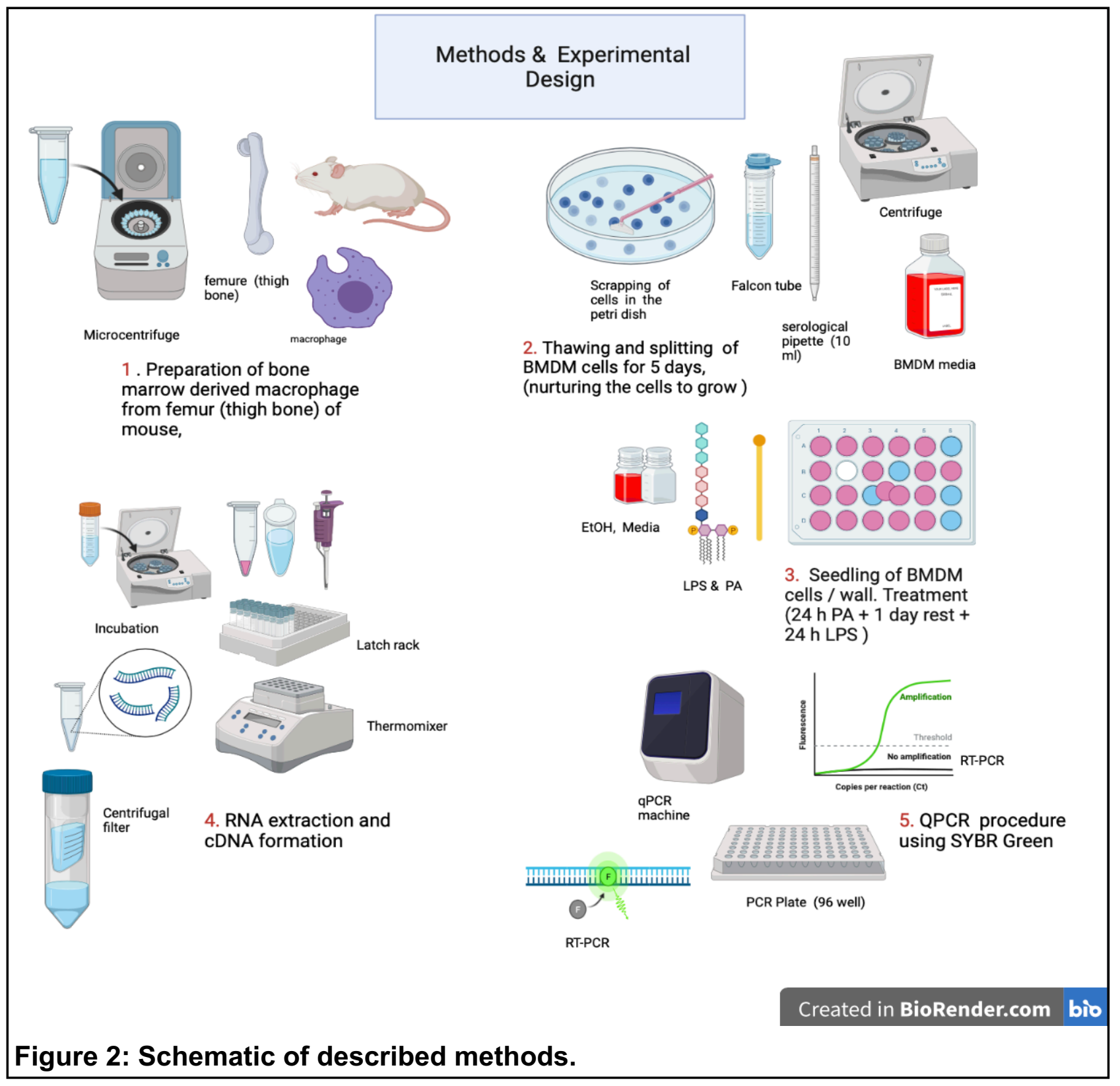




\section{E. RESULTS:}

Palmitic acid (PA) upregulates glycolysis in macrophages: To understand if PA is inducing glycolysis during trained immunity, we first induced trained immunity and then measured the expression of key glycolytic enzymes. The upregulation of key glycolytic enzymes can inform us if PA can induce glycolysis during trained immunity.

Thus, we first treated primary bone marrow-derived macrophages (BMDMs) with and without $1 \mathrm{mM}$ of PA for $24 \mathrm{~h}$, then subsequently treated with LPS $(10 \mathrm{ng} / \mathrm{mL})$ for an additional $24 \mathrm{~h}$. After these two treatments, we isolated mRNA from the BMDMs and measured expression of key glycolytic enzymes $h k 2$, glut1, and pfkp using qualitative Real-Time PCR. We found that all 3 glycolytic enzymes were expressed to significantly higher levels in LPS treated macrophages, as previously published (Fig 3 A-C), (Tannahill, et al., 2013). Interestingly, we found that glut1 was significantly up-regulated in PA-LPS treated macrophages (Fig 3B), suggesting that the expression of glut1 is upregulated during the PA-dependent trained immunity response. However, both $h k 2$ and pfkp were not found to be significantly up-regulated in the PA-LPS treated BMDMs (Fig 3 $A, C)$. These data suggest there is a bifurcation in regulation of these 3 glycolytic enzymes during PA-induced trained immunity, and that glut1 is positively regulated by the PAdependent trained immune response.

Next, we worked to understand the persistence of this PA-dependent increase in glycolysis during trained immunity. To do this, we treated BMDMs and without $1 \mathrm{mM}$ of PA for $24 \mathrm{~h}$, rested for $24 \mathrm{~h}$ with media only then subsequently treated with LPS (10 $\mathrm{ng} / \mathrm{mL})$ for an additional $24 \mathrm{~h}$. We found that BMDM treated with $1 \mathrm{mM}$ of PA followed by $24 \mathrm{~h}$ rest did not show significantly higher expression of any of the 3 glycolytic enzymes (Fig 3 D$F$ ). These data suggest that PA induces the expression of some glycolytic enzymes during trained immunity, but if the macrophages are rested for $24 \mathrm{~h}$ after the PA treatment, this allows the macrophage to recover to homeostatic expression of glycolytic enzymes. These data highlight the plasticity of the PA-dependent glycolytic influx during the trained immune response in macrophages. 


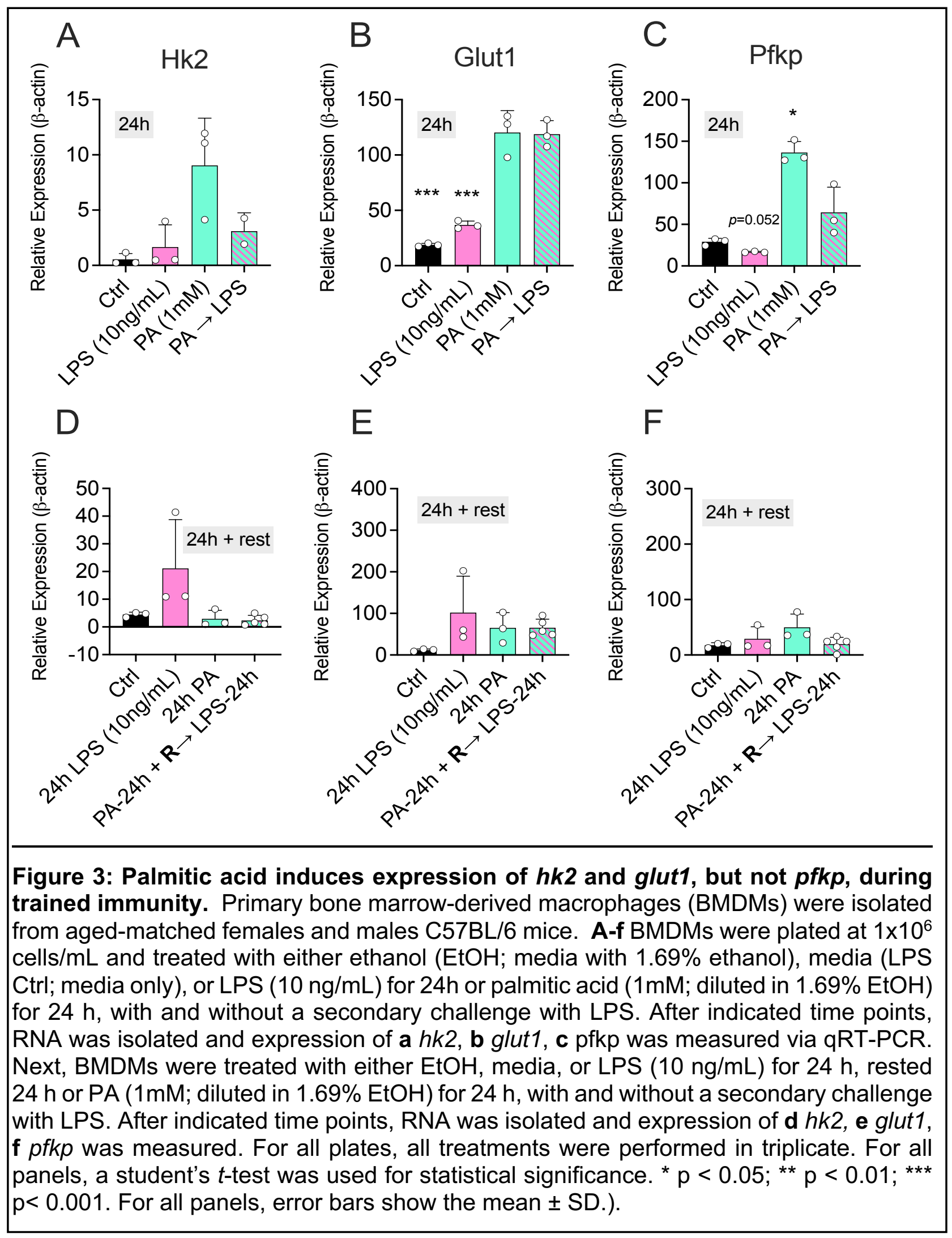




\section{F. DISCUSSION:}

Summary: We have found that PA may induce glycolysis by enhancing the expression of glycolytic gene: glut1 (Fig 3). However, the treatment of bone marrow-derived macrophages with $24 \mathrm{~h}$ PA, $24 \mathrm{~h}$ rested (Fig 3. D-F) and exposed to a secondary inflammatory stimulus (LPS), no longer upregulated glut1 expression (Fig 3). This suggests that resting of macrophages with only media for an additional $24 \mathrm{~h}$ can potentially impact on expression of glycolytic genes in glycolysis metabolisms. Overall, these data indicates that PA treatment of macrophages not only increases cytokine expression (Fig. 1), after exposure to LPS, but that it also confers an increase expression of some glycolytic enzymes. Our data support previously reported findings that high-fat diets containing PA appear to play an important role in the metabolic regulation of inflammatory diseases such as sepsis (Palsson-McDermott, at al., 2011, Napier et al., 2019). Specifically, the enhancement of LPS-induced glycolysis by PA occurs in conjunction with expression of pro-inflammatory cytokines (Fig 2-3).

Although trained immunity provides protection against a secondary non-specific infection, innate immune cell memory can also impair immune function, as observed in septic immune paralysis (Netea, el al., 2020) or persistent inflammatory activation evident in atherosclerosis (Netea, et al., 2020). Additionally, trained immunity is associated with an upregulation of several cellular metabolic pathways, including glycolysis and glutamine metabolism (Bekkering el al. 2018). Together, this data suggests that trained immunity is a double-edged sword, where it can be beneficial for resistance to infection, but detrimental in the context of inflammatory diseases (A.R. DiNardo, et al., 2021). Thus, PA-induced trained immunity may aggravate tissue damage during inflammatory disease and may contribute to obesity-induced metabolic disorder (Charles-Messance, et al., 2020). Our future studies will focus on identifying additional metabolic pathways and cellular mechanisms that are targeted by PA-treatment that lead to enhanced glycolysis and inflammation in the trained immunity response. These data will shed light on the potentially beneficial and dangerous effects of PA-induced trained immunity by PA- 
regulated metabolic alterations. 2-DG suppresses the LPS-induced succinate increase in macrophages so that the inhibition of IL-1 $\beta$ induction by 2-DG was observed, as a result, the inhibition of hexokinase by 2-DG is potentially useful for treating inflammatory disorders. In our study, this enzyme is inhibited without inhibitors treatments.

In conclusion, further data is essential in understanding the metabolic regulation of macrophages during PA-induced trained immunity which will be important for elucidating the mechanism of cellular regulation and hyper-inflammation in innate immune cells during inflammation. Palmitic acid is not only up-regulating trained immunity inflammation in macrophages and enhancing severity of inflammatory disease such as sepsis, but it can also impact on metabolism and leads to metabolic disorders in patients.

\section{G. REFERENCES:}

G. Arango Duque and A. Descoteaux, Macrophage cytokines: involvement in immunity and infectious diseases. Frontiers in immunology, 5, 491 (2014).

S. Bekkering, et al., Metabolic induction of trained immunity through the mevalonate pathway. Cell 172.1-2: 135-146 (2018).

S. Bekkering et al., Oxidized low-density lipoprotein induces long-term proinflammatory cytokine production and foam cell formation via epigenetic reprogramming of monocytes. Arteriosclerosis Thromb Vasc Biol 34, 17311738 (2014).

H. Charles-Messance, K. A. J. Mitchelson, E. De Marco Castro, F. J. Sheedy, H. M. Roche, Regulating metabolic inflammation by nutritional modulation. J Allergy Clin Immunol 146, 706-720 (2020).

S.C. Cheng, J. Quintin, RA. Cramer, et al., mTOR- and HIF-1a-mediated aerobic glycolysis as metabolic basis for trained immunity. Science 345.6204 (2014).

A. Christ, M. Lauterbach, and F. Latz, Western diet and the immune system: an inflammatory connection. Immunity 51.5: 794-811 (2019).

A. R. DiNardo, M. G. Netea, D. M. Musher, Postinfectious Epigenetic Immune Modifications - A Double-Edged Sword. N Engl J Med 384, 261-270 (2021). 
M. Divangahi et al. Trained immunity, tolerance, priming and differentiation: distinct immunological processes. Nat Immunol 22, 2-6 (2021).

J. Domínguez-Andrés, LAB. Joosten, M.G. Netea, Induction of Innate immune memory: the role of cellular metabolism, Current opinion in Immunology, 56: 1016 (2019).

E. Kaufmann et al., BCG Educates Hematopoietic Stem Cells to Generate Protective Innate Immunity against Tuberculosis. Cell 172, 176-190 e119 (2018).

B. Kelly, LA O'Neill, Metabolic reprogramming in macrophages and dendritic cells in innate immunity. Cell research 25.7: 771-784 (2015).

J. Kleinnijenhuis et al., Bacille Calmette-Guerin induces NOD2-dependent nonspecific protection from reinfection via epigenetic reprogramming of monocytes. PNA 109, 17537-17542 (2012).

J. Korbecki, K. Bajdak-Rusinek, The effect of palmitic acid on inflammatory response in macrophages: an overview of molecular mechanisms. Inflamm Res 68, 915-932 (2019).

P.S. Laine, et al., Palmitic acid induces IP-10 expression in human macrophages via NF-kB activation. Biochemical and biophysical research communications 358.1:150-155 (2007).

X. Liu, MA Boyer, AM Holmgren, S Shin, Legionella-infected macrophages engage the alveolar epithelium to metabolically reprogram myeloid cells and promote antibacterial inflammation. Cell Host \& Microbe 28.5: 683-698(2020).

CJ. Martin, KN Peters, SM Behar, Macrophages clean up: efferocytosis and microbial control. Current opinion in microbiology 17: 17-23 (2014).

M. Matsuura, Structural modifications of bacterial lipopolysaccharide that facilitate gram-negative bacteria evasion of host innate immunity. Frontiers in immunology 4:109(2013).

T.H. Mogensen, Pathogen Recognition and Inflammatory Signaling in Innate Immune Defenses, Clinical microbiology reviews, 22(2), 240-273 (2009). 
K. Murphy, C. Weaver, C. Janeway, Janeway's immunobiology, New York, $9^{\text {th }}$ edition, Garland Science, (2017).

B.A. Napier, et al., Western diet regulates immune status and the response to LPS-driven sepsis independent of diet-associated microbiome, Proceedings of the National Academy of Sciences 116.9: 3688-3694 (2019).

M.G. Netea et al., Defining trained immunity and its role in health and disease. Nat Rev Immunol 20, 375-388 (2020).

M.G Netea, Training innate immunity: the changing concept of immunological memory in innate host defence, European journal of clinical investigation 43.8: 881-884 (2013).

M.G, Netea, J Quintin, JWM Van Der Meer. Trained immunity: a memory for innate host defense. Cell host \& microbe 9.5: 355-361(2011).

EM. Palsson-Mcdermott et al., Pyruvate Kinase M2 Regulates Hif-1a Activity and IL-1b Induction and Is a Critical Determinant of the Warburg Effect in LPSActivated Macrophages, Cell Metabolism 21.1: 65-80 (2015).

EM. Palsson-McDermott, LA. O'Neill, The Warburg effect then and now: from cancer to inflammatory diseases." Bioessays 35.11: 965-973 (2013).

S. Saeed et al., Epigenetic programming of monocyte-to-macrophage differentiation and trained innate immunity. Science 345, 1251086 (2014).

E. A. Schwartz et al., Nutrient modification of the innate immune response: a novel mechanism by which saturated fatty acids greatly amplify monocyte inflammation. Arterioscler Thromb Vasc Biol 30, 802-808 (2010).

AL. Seufert et al., in preparation, Dietary saturated fatty acids mediate innate immunity memory that enhances severity of acute septic shock and clearance of infection. The Preprint services for Biology, (2021).

G.M. Tannahill, et al., Succinate is an inflammatory signal that induces IL-1 $\beta$ through HIF-1a. Nature 496.7444: 238-242 (2013).

H.T. Tzeng, I.T. Chyuan, W.Y. Chen, Shaping of innate immune response by fatty acid metabolite palmitate. Cells 8.12: 1633 (2019). 
A. Viola, F. Munari, et al., The metabolic signature of macrophage responses. Frontiers in immunology, 10, 1462, (2019).

F. Wang, et al., Interferon gamma induces reversible metabolic reprogramming of M1 macrophages to sustain cell viability and pro-inflammatory activity. EBioMedicine 30: 303-316 (2018).

L. Yang, et al., PKM2 regulates the Warburg effect and promotes HMGB1 release in sepsis. Nature communications 5.1:1-9 (2014).

L. Zhu, Q. Zhao, T. Yang, et al., Cellular metabolism and macrophage functional polarization. International reviews of immunology, 34(1), 82-100 (2015). 